\title{
Functions of polyphenols and its anticancer properties in biomedical research: a narrative review
}

\author{
Pritam Bhagwan Bhosale", Sang Eun Ha", Preethi Vetrivel, Hun Hwan Kim, Seong Min Kim, \\ Gon Sup Kim
}

Research Institute of Life Science and College of Veterinary Medicine, Gyeongsang National University, Jinju, Korea

Contributions: (I) Conception and design: GS Kim, SM Kim, PB Bhosale; (II) Administrative support: GS Kim; (III) Provision of study materials or patients: All authors; (IV) Collection and assembly of data: All authors; (V) Data analysis and interpretation: PB Bhosale; (VI) Manuscript writing: All authors; (VII) Final approval of manuscript: All authors.

\#These authors contributed equally to this work.

Correspondence to: Seong Min Kim; Gon Sup Kim. Research Institute of Life Science and College of Veterinary Medicine, Gyeongsang National University, Jinju 52828, Korea. Email: ksm4234@naver.com; gonskim@gnu.ac.kr.

\begin{abstract}
Cancer is a major health concern as the incidence is growing worldwide and still lacks successful therapies. Plant-derived functional foods are getting considerable attention, primarily due to their safety and therapeutic potential. Polyphenols are a group of mostly natural, water-soluble organic compounds. Here, we review the functions of selected polyphenols and their anticancer properties on numerous cancer cell lines and their mechanisms. The literature search was performed using the electronic database PubMed, Google scholar up to June 2020, with the following keywords-polyphenol, polyphenol anticancer, quercetin anticancer, resveratrol anticancer, curcumin anticancer, and kaempferol anticancer. Chemical structures of the selected polyphenols were obtained using the ChemDraw program. The initial search identified 40,554 polyphenols papers and among that, 2,559 were limited to polyphenol and cancer, 987 quercetin and cancer, 2,174 curcumin and cancer, 1,079 resveratrol and cancer, and 226 were limited to kaempferol and cancer. A total of 84 papers are included in this review paper. Most studies report the multiple anticarcinogenic properties of plant-derived polyphenols, including its inhibitory effects on the proliferation of cancer cells, tumor expansion, angiogenesis, inflammation, and metastasis. Besides, some studies shows potential synergistic effects when polyphenol treatment combined with chemotherapeutic agents. Anticancer effects of polyphenolic compounds like quercetin, curcumin, resveratrol, and kaempferol are investigated on numerous cancer cell lines and have shown prominent results. The present review provides a direction to determine the anticarcinogenic ability of the selected polyphenols in vitro and in vivo. Consequently, the use of polyphenols in cancer treatment should be investigated in-depth in the future.
\end{abstract}

Keywords: Anticancer effect; curcumin; kaempferol; polyphenols; quercetin; resveratrol

Submitted Jun 19, 2020. Accepted for publication Nov 06, 2020.

doi: $10.21037 /$ tcr-20-2359

View this article at: http://dx.doi.org/10.21037/tcr-20-2359

\section{Introduction}

Cancer is described as a series of complex processes involving impaired cell death, unregulated cell proliferation, and changes in cell physiology, which often leads to malignant tumor formation resulting in metastasis being invaded by distant tissues (1). The incidence of cancer and mortality is rising rapidly around the world. According to GLOBOCON, an estimation of 18.1 million new cancer cases and 9.6 million cancer-related deaths cancer has been reported in 2018 (2). The causes are complex but focus on both population growth and aging as well as shifts in the prevalence and distribution of major cancer risk factors, including socio-economic development (3). Multi-stage carcinogenesis is a commonly accepted concept 
of cancer development and is divided functionally into three stages: initiation, promotion, and progression $(4,5)$. Current therapies such as chemotherapy and radiotherapy have drawbacks, including drug resistance in cancers to anticancer medications and adverse radiotherapy outcomes $(6,7)$.

Over the past centuries, pharmacological systems all over cultures have professed and supported the use of edible substances, particularly plant-derived ones, to treat and prevent diseases, thereby creating knowledge of the potential of natural agents known as phytochemicals as anticarcinogenic agents for cancer $(8,9)$. Almost $47 \%$ of the anticancer drugs available on the market today are derivatives of natural products (10). Phenolic compounds, such as flavonoids, are the most effective secondary plantderived metabolites for cancer treatment $(11,12)$.

Natural polyphenols are organic chemicals derived from plants, distinguished structurally having two or more phenol units (13). Numerous studies have been carried out on polyphenols to examine their possible health benefits, involving defense against oxidative stress, diabetes, cardiovascular disease, neurodegenerative disease, and aging (14). These polyphenols can exert anticancer effects through a broad range of mechanisms, that includes cancer cell removal by modification of signaling pathways, inhibition of cell cycle events, and apoptosis induction. Polyphenols also regulate the activities of enzymes involved in tumor cell proliferation. Recent studies are implicating natural polyphenols and their anti-cancer potential through a wide range of properties, e.g., antiangiogenic, antimetastasis, DNA-interaction, and others $(15,16)$.

In this review, we summarize selected examples from the recent literature to show the potential of naturally derived polyphenols as anticancer agents.

\section{Classification of polyphenols}

Polyphenols are listed according to the number of phenol rings and the structural elements that bind them together. The classified groups include phenolic acids, stilbenes, lignans, and flavonoids. Flavonoids have antioxidant and anti-inflammatory properties, which are found in legumes, fruits, vegetables, green tea, and red wine. They are subdivided into six classes: anthocyanins, flavonols, flavones, flavanones, isoflavones, and flavanols $(13,17)$. Flavonols are the most ubiquitous flavonoids that are abundant in fruits and leafy vegetables (14). The key members are quercetin and kaempferol, and the most abundant sources include onions, curly kale, leeks, and broccoli (17). Flavones consists chiefly of glycosides of luteolin and apigenin (16). Flavanones are found in citrus, tomatoes, and other aromatic plants like mint. Isoflavones are flavonoids possessing structural similarities with estrogens and are mostly found in leguminous plants. The primary source of isoflavones in the human diet is soya and its processed products. Among phenolic acids, hydroxybenzoic acids are found in tea, cinnamon, coffee, kiwis, blueberries, apples, plums, and cherries (14). Stilbenes are found in meager quantities in the human diet. Resveratrol is one of the wellstudied polyphenols for anticarcinogenic effects in medicinal plants (18). Lignans are found in flax seeds, grains, legumes, cereals, algae, fruits, and certain vegetables (19). Anthocyanins occur in all tissues of plants, including stems, leaves, flowers, roots, and fruits. Flavanols are found in different types of fruits, chocolate, and green tea (17). The overall classification of polyphenols is illustrated in Table 1.

\section{Polyphenols as an anticancer agent}

The relationship between polyphenol consumption and cancer risk occurrence is regularly assessed and metaanalyzed over the last $10-15$ years. In a meta-analysis of prospective studies, isoflavone intake was associated with a $19 \%$ reduction in the risk of gastric cancer (20). Recent epidemiologic studies have indicated a decrease in breast cancer risk by soy product consumption (21). The consumption of isoflavone and flavonol was correlated with an estimated $30 \%$ reduction in the incidence of ovarian and endometrial cancers across cohort and casecontrol studies (22). Intake of soy isoflavones and soy-based foods associated with reduced risk of colorectal cancer in two Asian population meta-analyses, one showing a $23 \%$ reduction in risk from 13 case-control studies and four prospective studies. Also, a high intake of total soy products was linked to a reduced risk of colorectal cancer in a casecontrol Korean survey, particularly at the distal and rectal sites (22). A meta-analysis of green tea polyphenols showed a $19 \%$ reduction in the risk of prostate cancer only when seven or more cups of green tea were ingested daily (23). A case-control study in Canada reported beneficial effects of a high dietary intake of total flavonoids on lung cancer risks $(24,25)$. Overall, polyphenols compounds are attractive molecules for cancer treatment, and some of the most studied polyphenols are described briefly in the following sections. We present the following article in accordance with the Narrative Review reporting checklist (available at 
Table 1 Classification of polyphenols

\begin{tabular}{|c|c|}
\hline Classification & Representative members \\
\hline \multicolumn{2}{|l|}{ Flavonoids } \\
\hline Anthocyanins & Delphinidin, pelargonidin, cyanidin, malvidin, aurantinidin, peonidin, petunidin, rosinidin \\
\hline Flavanols & Epicatechin, epigallocatechin, EGCG, procyanidins, prodelphinidins \\
\hline Flavanones & $\begin{array}{l}\text { Hesperidin, naringenin, butin, eriodictyol, homoeriodictyol, isosakuranetin } \\
\text { Naringin, pinocembrin, poncirin, sakuranetin } \\
\text { Sakuranin, sterubin, pinostrobin }\end{array}$ \\
\hline Flavones & $\begin{array}{l}\text { Apigenin, chrysin, luteolin, acacetin, genkwanin, echioidinin, baicalein, oroxylon, wogonin, geraldone, tithonine, } \\
\text { diosmetin, pilloin, velutin, scutellarein, hispidulin, sorbifolin, pectolinarigenin, zapotin, tricetin, jaceosidin, } \\
\text { sinensetin, onopordin, tangeretin }\end{array}$ \\
\hline Flavonols & $\begin{array}{l}\text { Quercetin, kaempferol, myricetin,isorhamnetin, galangin, azaleatin, fisetin, gossypetin, kaempferide, morin, } \\
\text { natsudaidain, pachypodol, rhamnazin }\end{array}$ \\
\hline \multicolumn{2}{|l|}{ Phenolic acids } \\
\hline Hydroxybenoic acid & Ellagic acid, gallic acid, syringic acid \\
\hline Hydroxycinnamic acid & Ferulic acid, chlorogenic acid, caffeic acid \\
\hline Lignans & $\begin{array}{l}\text { Sesamin, secoisolariciresinol diglucoside, pinoresinol, lariciresinol, syringaresinol, hydroxymatairesinol, } \\
\text { matairesinol }\end{array}$ \\
\hline Stilbenes & Resveratrol, pterostilbene, piceatannol, viniferins \\
\hline
\end{tabular}

http://dx.doi.org/10.21037/tcr-20-2359).

\section{Methods}

The literature review was done using the electronic database PubMed and Google scholar up to June 2020, with the following keywords: polyphenol, polyphenol anticancer, quercetin anticancer, resveratrol anticancer, curcumin anticancer, and kaempferol anticancer. The search did not include editorials, letters, comments, and conference letters. Paper search was limited to the English language. All data were extracted in a standard pre-determined format, including information on first author's name, publication year, polyphenols, and its anticancer effects on different cell lines. The articles included in the study were full-length original articles and focused on various cancer cell lines. Chemical structures of the polyphenols are provided using the ChemDraw program.

\section{Results}

The process of the study selection was shown in Figure 1 . Of the 40,554 polyphenol papers were found, 2,559 were limited to polyphenol and cancer, 987 quercetin and cancer, 2,174 curcumin and cancer, 1,079 resveratrol with cancer, and 226 were limited to kaempferol and cancer. A total of 84 papers were selected for discussion in this review paper. The chosen articles included original research articles. The data about the anticancer effect of polyphenol on different cancer cell lines are extracted and summarized in Table 2.

\section{Discussion}

\section{Quercetin}

The flavonol quercetin (3,3',4',5,7-pentahydroxyflavone; Figure 2) is one of the most copious naturally occurring polyphenols. Quercetin is found in several plant parts (leaves, grains, and fruits) as well as in foods and beverages, including tea and red wine (26,27). Quercetin is identified to have a wide range of biological activities, including antioxidant, antimicrobial, anticarcinogenic, antidiabetic activities, and anti-inflammatory. Quercetin functions as an antioxidant at low concentrations and induces chemopreventive action. However, high levels of quercetin act as a pro-oxidant and may cause a chemotherapeutic effect $(28,29)$. The recommended regular doses of quercetin 


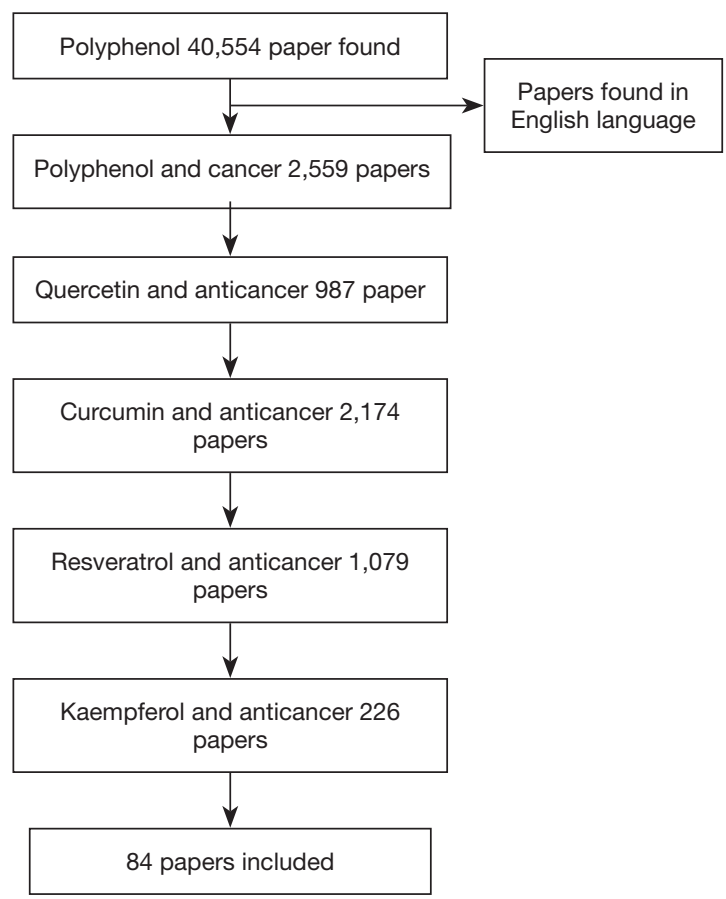

Figure 1 Summary of search strategy employed.

in dietary supplements are typically in the range of up to $1,000 \mathrm{mg}(30)$.

Growth inhibition by quercetin and antineoplastic drug 5-fluorouracil (5-FU) evaluated in HepG2 and SMCC-7721 (human hepatocellular carcinoma cell line). Dose-dependent cell growth inhibition by quercetin in both the cell lines and increased 5-FU efficacy. Furthermore, quercetin modifies the expression of apoptosis-dependent proteins, reduces tumor growth, and increases 5-FU output in the mouse xenograft model (31). In another study, quercetin-treated MCF-7 (human breast cancer cell line) cells showed dose- and timedependent decreased proliferation and apoptosis induction by Bax upregulation and Bcl-2 downregulation (32). Quercetin induced apoptosis and inhibits hypoxia-induced 5 ' adenosine monophosphate-activated protein kinase (AMPK) activity in HCT116 (human colon cancer cell line) cells (33). Quercetin-treated BGC-823 (human gastric cancer cell line) cells displayed morphological changes like loss of attachment, chromatin condensation, shrinkage, cell rounding, and condensed nucleus (34). On the other hand, BC3, BCBL1, and BC1 (primary effusion lymphoma cell line) cells induced apoptosis treated with quercetin inhibits phosphoinositide 3-kinases/serine/threonine-specific protein kinase/mammalian target of rapamycin (PI3K/AKT/mTOR) pathway, $W n t / \beta$-catenin pathway and signal transducer and activator of transcription 3 (STAT3) activation and autophagy in PEL cells (35). Hela (cervical cancer cell line) cells showed inhibited proliferation, induced autophagy by LC3B-I conversion into LC3B-II in a concentrationdependent way, and apoptosis significantly promoted by quercetin and autophagy inhibitors (36). Similarly, quercetin inhibited cell viability, the early apoptotic population in a dose-dependently, and G2/M phase cell cycle arrest in LM3 (human hepatocellular carcinoma cell line) cells. Further, it inhibited the activation of the Janus kinase 2 (JAK2)/STAT3 pathway, migration, and invasion, reduced tumor growth, and induced autophagy by overexpression of LC3B and decreased p62 (37).

Quercetin decreases cell viability in PATU-8988 and PANC-1 (pancreatic cancer cell line) with the expression of neural cadherin (N-cadherin), epithelial cadherin (E-cadherin), vimentin, hinders migration and invasion, reverses interleukin 6 (IL-6)-induced increase in malignant pancreatic cells by inhibiting the signaling pathway STAT3 (38). The exposure to HUVEC (human umbilical vein endothelial cell line) and PC-3 (human prostate cancer androgen-independent cell line) cells to quercetin caused dose-dependent and time-dependent decrease in proliferation. Further, quercetin inhibited the migration and invasion, angiogenesis, and tumor tissue in xenograft models (74). Similarly, quercetin also reduced proliferation, migration, and invasion, induced apoptosis, and blocked angiogenesis by targeting vascular endothelial growth factor (VEGF) in Y79 cells (human retinoblastoma cell line) (75). RF/6A (Rhesus choroids-retina endothelial cell line) cells treated with quercetin inhibited proliferation, cell migration, restrained tube formation in Matrigel assay, increased apoptotic rate slightly, and inhibited angiogenesis (76). Quercetin inhibits MCF-7 (human breast cancer cell line) xenograft growth in mice, demonstrating the antitumor and antiangiogenic effect of quercetin on breast cancer in vivo and inhibited angiogenesis via activating the calcineurin/nuclear factor of activated T-cells (NFAT) pathway (77). Apoptosis was induced in HT29 (human colon adenocarcinoma cell line) and CT26 (mouse colon carcinoma cell line) after quercetin treatment. CT26 cells shown activated extracellular signal-regulated kinases (ERK), c-Jun N-terminal kinases (JNK), and p38 mitogenactivated protein kinases (MAPK) signaling pathways, inhibit migration and invasion by controlling the expression of E-cadherin, $\mathrm{N}$-cadherin, $\beta$-catenin, snail, and in vivo metastasis (39). These studies indicate that quercetin has profound anticancer effects, based on its ability to suppress 
Table 2 In vitro and in vivo anti-cancer effect of selected polyphenols

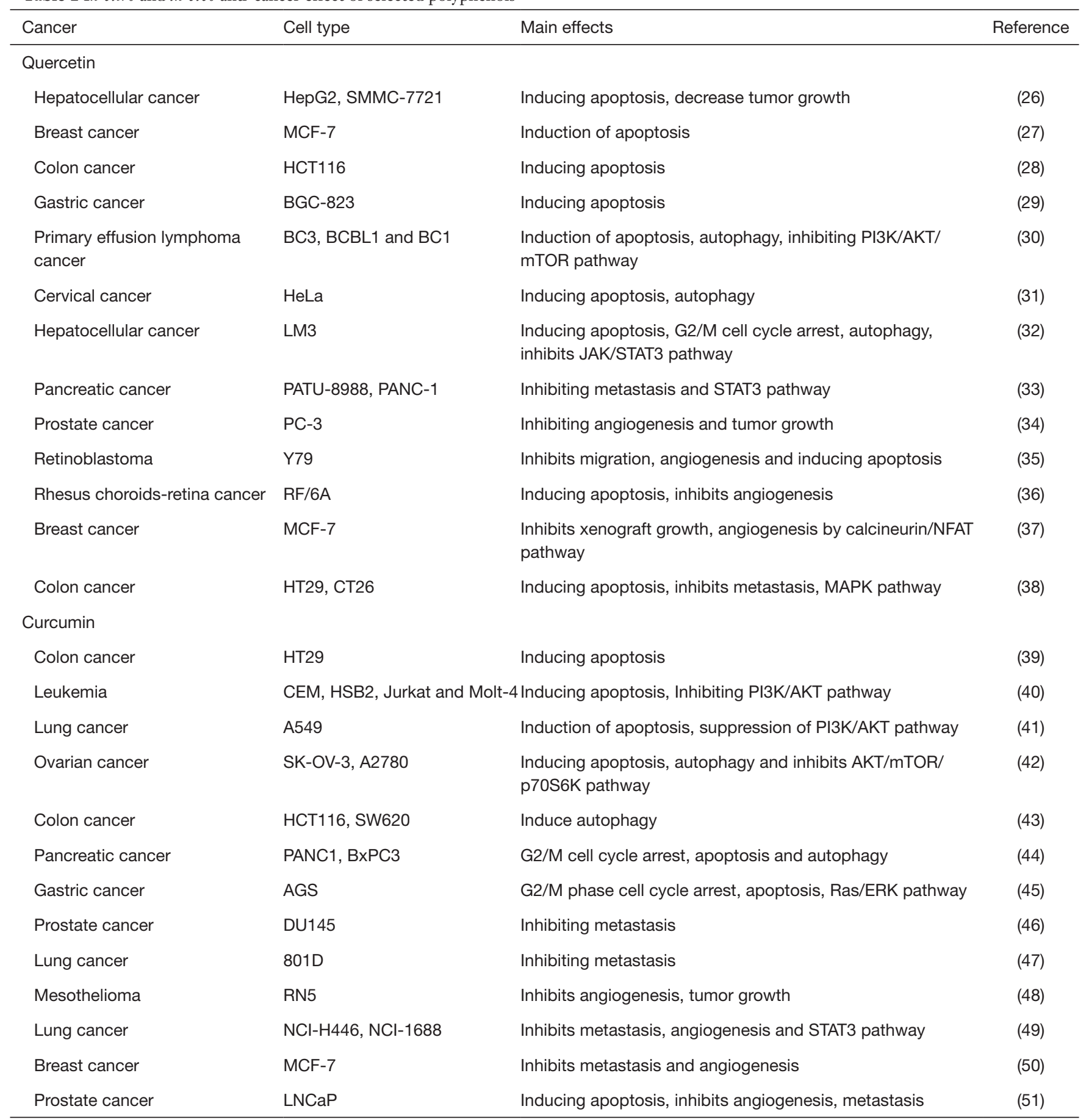

Table 2 (continued) 
Table 2 (continued)

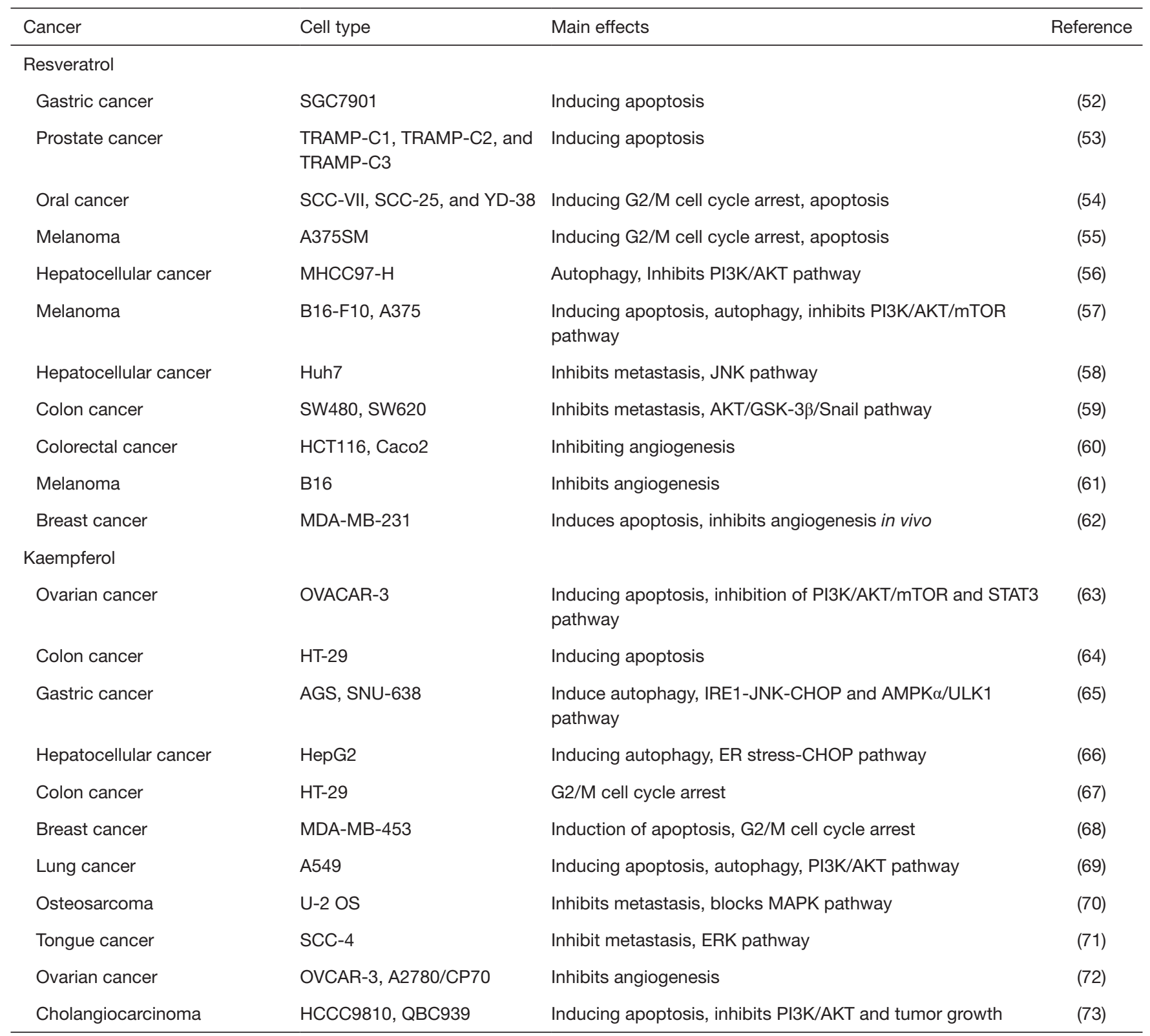

proliferation, induce apoptosis, trigger cell cycle arrest in numerous cancer cell lines, and can be used as a potent anticancer agent.

\section{Curcumin}

Curcumin [1,7-bis(4-hydroxy-3-methoxyphenyl)-1,6heptadiene-3,5-dione] is a hydrophobic polyphenol (Figure 3), bright-yellow, present in the rhizome of Curcuma longa, a perennial herb in the family Zingiberaceae (40). Curcumin belongs to a chemical class of polyphenols has been shown to have therapeutic benefits in several chronic diseases including arthritis, neurodegenerative diseases, metabolic syndrome, liver disease, obesity, inflammation, and in several cancer types (41). Curcumin shown to have significant antiinflammatory, antioxidant, anticoagulant, antimutagenic, anticarcinogenic, and anti-infective effects. Curcumin has also demonstrated significant healing properties for wounds 
<smiles>O=c1c(O)c(-c2ccc(O)c(O)c2)oc2cc(O)cc(O)c12</smiles>

Figure 2 Chemical structure of quercetin.

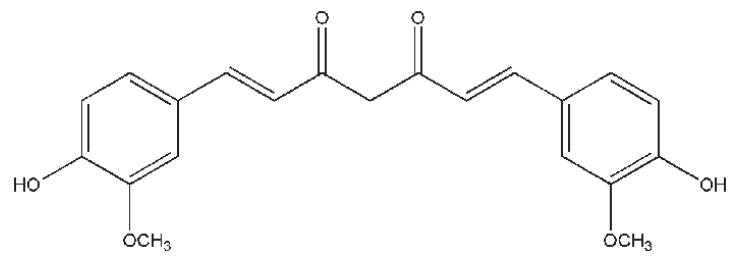

Figure 3 Chemical structure of curcumin.

$(42,43)$. Since then, the scientific interest in the use of curcumin for health benefits has increased.

The recent study indicated that curcumin could suppress proliferation significantly and induce apoptosis in HT-29 (human colon cancer cell line) cells through the mitochondrial cell death pathway. Curcumin decreases, Bcl-xL/Bad, Bcl-2/Bax ratios, preceded by caspase-3 activation (44). CEM, HSB2, Jurkat, and Molt-4 (human T-leukemia cell lines) cells treated with curcumin caused dose-dependent growth inhibition, induce apoptosis, inhibit PI3K/AKT, and release of cytochrome c, poly (ADPribose) polymerase (PARP), and cleaved caspase-3 (45). Similarly, curcumin exposure to A549 cells (human nonsmall lung cancer cell line) prevented cell proliferation, induced apoptosis, elevated caspase-3 activity, upregulation of miR192-5p, and suppression of the PI3K/AKT signaling (46). Curcumin repressed proliferation of SKOV-3 and A2780 cells (human ovarian cancer cell lines), stimulated apoptosis-induced autophagy with many autophagic vesicles and increased expression of Atg3, Beclin, and LC3B-II protein. Further, it inhibited the activity of the AKT/mTOR/p70S6K signaling pathway (47). Curcumintreated HCT116 and SW620 cells (human colon cancer cell lines) showed growth inhibition followed by autophagy induction with LC3B, P-62 protein regulation, yesassociated protein 1 (YAP) suppression and can reverse the effect of YAP on colon cancer cells (48). Curcumin-exposed PANC1 and BxPC3 cells (human pancreatic cell lines) showed suppressed cell growth, triggered G2/M phase cell arrest, autophagy, and apoptosis (49). Similarly, cell growth of curcumin-treated AGS cells (human gastric cancer cell line) was inhibited, caused apoptosis and G2/M phase cell cycle arrest with the Ras/ERK signaling pathway (50).

DU145 cells (human prostate cancer cell line) exposed to curcumin reported growth inhibition in a dose-dependent manner. Additionally, curcumin inhibited migration, matrix metalloproteinase (MMP-2) expression, then inhibited metastasis in a prostate cancer cell line (51). Likewise, Curcumin-treated 801D (human lung cancer cell line) cells suppressed cell growth and can effectively inhibit epidermal growth factor (EGF) or transforming growth factor (TGF- $\beta 1$-induced migration, invasion, and the Rac1dependent signaling pathway. Curcumin also inhibited cell invasion through MMP-2 and MMP-9 expression in vivo (78). RN5 cells (murine malignant mesothelioma cell line) exposed to curcumin cause growth inhibition in a concentration-dependent manner. Further, it decreases tumor growth in vivo, and then angiogenesis was confirmed by immunostaining (79). On the other hand, NCI-H446 and NCI-1688 (human lung cancer cell lines) treated with curcumin exhibited cell proliferation. Curcumin also inhibited the expression of MMP-2, MMP-7, VEGF, Bcl-XL, Survivin, and ICAM-1 in cells confirming the inhibition migration, angiogenesis, and invasion through suppression of the STAT3 (80). Comparative study of BDMC-A (an analog of curcumin) and curcumin-treated MCF-7 (human breast cancer cell line) cells shown significant downregulation of marker genes related to angiogenesis, metastasis, and invasiveness (52). In vivo experiments have shown that curcumin sensitizes TRAILresistant LNCaP cells (human prostate cancer cell line) through various mechanisms. It stimulates death receptors, upregulates proapoptotic Bax members, Bak, suppresses antiapoptotic Bcl-xL proteins, and further inhibits VEGF, MMP-2, MMP-9 activation, which plays a vital role in metastasis, invasion, and angiogenesis (53). These research studies confirm that curcumin has a strong anticancer effect on the diverse cancer cell lines, and it can be considered in drug development for cancer treatment.

\section{Resveratrol}

Resveratrol (3,5,4'-trihydroxystilbene; Figure 4), a polyphenolic compound which occurs naturally, is a stilbene found in berries, grapes, peanuts, and other plant sources (54). Resveratrol have antioxidative, cardio- 


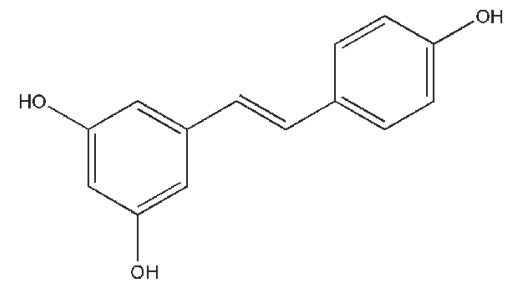

Figure 4 Chemical structure of resveratrol.

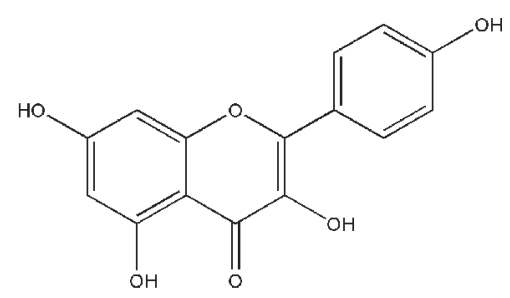

Figure 5 Chemical structure of kaempferol.

protective, estrogenic/anti-estrogenic, anti-inflammatory and antitumor properties (55). This compound is also well known for its anticancer effects. Also, several laboratory studies have shown that resveratrol supports a wide variety of diseases, including cardiovascular disorders (CVDs), diabetes, obesity, cancers, hepatic diseases, Parkinson's disease, and Alzheimer's disease. Resveratrol has shown protective effects on a variety of cancers such as breast, prostate, colorectal, lung, ovarian, cervical, hepatic, and gastric cancer (56).

Resveratrol suppresses the dose-dependent growth of cells with SGC7901 (human gastric adenocarcinoma cell line) and induces apoptosis with increased reactive oxygen species (ROS) levels. Resveratrol treatment to SGC7901 cells caused DNA damage with higher $\gamma$-H2AX levels and reduced ku70 in western blot analysis (57). TRAMP-C1, TRAMP-C2, and TRAMP-C3 (murine prostate cancer cell lines) cells treated with resveratrol caused mitochondrialmediated caspase-dependent apoptosis and increased expression of $\gamma-\mathrm{H} 2 \mathrm{AX}$ by sensitizing DNA damage (58). SCC-VII, SCC-25, and YD-38 (oral squamous cancer cell lines) cells treated with resveratrol inhibit cell growth, G2/M phase cell cycle arrest regulating the cell cycle proteins, and induces apoptosis (59). Similarly, resveratrol treated A375SM cells (human malignant melanoma cell line) reported the G2/M phase cell cycle arrest, elevated ROS generation, endoplasmic reticulum (ER) stress leading to apoptosis (60). MHCC97-H (human hepatocellular cancer cell line) cells exposed to resveratrol causes growth inhibition and mortality through autophagy induction with the expression of Beclin1, LC3B II/I, via regulating the $\mathrm{p} 53$ and PI3K/AKT pathways (61). Likewise, B16-F10 (murine melanoma cell line) and A375 (human melanoma cell line) cells treated with resveratrol showed reduced cell viability, induce apoptosis, and inhibits invasion and migration with increased cleaved caspase-9 levels in A375 cells. Resveratrol also induces autophagy in B16-F10 cells with increased expression of Beclin1 and LC3B-II/LC3B-I proteins and significantly reduced levels of p62 protein. Further, it inhibits the PI3K/AKT/mTOR pathway (62).

The cytotoxic effect of resveratrol evaluated on Huh7 (human hepatocellular cancer cell line) cells revealed reduced migration, invasion, and protein levels of urokinase plasminogen activator receptor (u-PA) in a concentration-dependent manner. Resveratrol can inhibit the phosphorylation and binding activities of JNK 1/2, causing down-regulation of expression of u-PA to prevent metastasis. Resveratrol exhibited an inhibitory effect on many essential metastasis stages (81). Resveratrol-treated cells SW480 and SW620 (human colon cancer cell lines) inhibits invasion and metastasis of colon cancer cells by reversing epithelial-mesenchymal transition (EMT) via the AKT/GSK-3 $\beta /$ Snail signaling pathway (82). Resveratrol exhibits a concentration-dependent inhibitory effect on HCT116 and Caco2 (human colorectal carcinoma cell lines) cells.

Further, it induces insulin expression, intrinsic and extrinsic apoptotic pathway decreases VEGF levels in treated cells. The enhancement of hypoxia-inducible factor $(\mathrm{HIF}-1 \alpha)$ protein expression resulted in the inhibition of VEGF expression (83). Likewise, B16 (murine melanoma cell line) cell treated with resveratrol together with antineoplastic drug fluorouracil inhibits migration, reduce VEGF levels, and angiogenesis (84). MDA-MB-231 (human breast adenocarcinoma cell line) tumors treated with resveratrol induces apoptosis and inhibits xenografts angiogenesis in vivo (63). In general, these studies indicate that resveratrol has anticancer properties that could be used as an effective anticancer agent.

\section{Kaempferol}

Kaempferol [3,5,7-trihydroxy-2-(4-hydroxyphenyl)-4H-1benzopyran-4-one; Figure 5], is extracted from tea and found in various widely known vegetables and fruits, including broccoli, beans, gooseberries, kale, strawberries, grapes, 
citrus fruits, brussel sprouts, tomatoes, grapefruits and apples (64). It has also been recognized in various medicinal plants as Acacia nilotica (L.), Aloe vera (L.), Crocus sativus (L.), Ginkgo biloba (L.), Hypericum perforatum (L.), Phyllanthus emblica (L.), Ribes nigrum (L.), and Rosmarinus officinalis (L.) (65). Kaempferol and its glycosylated derivatives are neuro-protective, cardio-protective, antidiabetic, anti-inflammatory, antitumor, antioxidant, antimicrobial, and have anticancer functions (66). Several edible plants contain kaempferol, and the human dietary intake of kaempferol is reported to be up to around $10 \mathrm{mg} /$ day (67).

Kaempferol triggers antiproliferative effects on OVACAR-3 cells (human ovarian cancer cell line) and affects cell viability. Further, apoptosis percentage also increased in a concentration-dependently supported by the regulation of apoptotic proteins like cleaved caspase-3 and cleaved caspase-9. Kaempferol triggers inhibition of PI3K/AKT/mTOR and STAT3 signaling (68). Similarly, kaempferol treatment triggers apoptosis in HT-29 cells (human colon cancer cell line) through both extrinsic and intrinsic pathways, changes the expression Bcl2 family proteins that lead to mitochondrial membrane depolarization and release cytochrome c from the mitochondria. In cytosol cytochrome c activates caspase-9, further allows caspase-3 activation (69). AGS and SNU-638 cells (human gastric cancer cell lines) exposed to kaempferol encouraged autophagy and cell death, by conversion of LC3B-I to LC3B-II, the decrease p62 via the activation of the IRE1-JNK-CHOP signaling, ER stress, and AMPKa/ ULK1 pathway (70). Kaempferol induced autophagy in HepG2 (human hepatocarcinoma cell line) due to increase expression of Atg5, Atg7, and Beclin1 proteins. It promoted a conversion from LC3B I to LC3B II in a doseand time-dependent manner via the ER stress- C/EBP homologous protein (CHOP) pathway (71). HT-29 (human colon cancer cell line) cell treatment with kaempferol inhibits proliferation, induces $\mathrm{G} 2 / \mathrm{M}$ cell cycle arrest by modulating the expression of cell cycle proteins timedependent manner (72). Likewise, kaempferol treatment to MDA-MB-453 (human breast cancer cell line) causes G2/ $M$-phase cell cycle arrest via downregulated CDK1 and apoptosis via modulation of the p53 pathway (73). A549 (human lung cancer cell line) cells treated with kaempferol showed inhibition of cell proliferation, promotes apoptosis, autophagy by increased expression of Beclin-1, and the ratio of LC3B-II/I and up-regulated the expression of phosphatase and tensin homolog (PTEN) and inactivated
PI3K/AKT pathway (85).

U-2 OS cells (human osteosarcoma cell line) exposed to kaempferol inhibits invasion, migration, and adhesion in a concentration-dependently. It further reduces activities of MMP-2, MMP-9, uPA, inhibits the DNA-binding activity of activator protein-1 (AP-1), and blocks the activation of MAPKs in U-2 OS cells (86). Similarly, kaempferol treated SCC-4 cells (human tongue squamous cell cancer cell line) suppressed cell invasion and migration in a dose-dependent manner. Furthermore, inhibits AP-1 activity, reduces MMP-2 expression, ERK1/2 phosphorylation, and showed antimetastasis potential (87). OVCAR-3 and A2780/CP70 (human ovarian cancer cell lines) treated with kaempferol inhibits VEGF expression, tumorigenesis, angiogenesis, and HIF- $1 \alpha$ protein expression in vivo (88). HCCC9810 and QBC939 (human cholangiocarcinoma cancer cell lines) treatment with kaempferol inhibits the proliferation, reduces colony formation, and induces apoptosis. Furthermore, it suppresses the migration, invasion, PI3K/ AKT pathway and affects the apoptosis- and invasionrelated proteins by inhibiting tumor growth (89). These studies suggest that kaempferol has a significant anticancer impact on various cancer cell lines, and further studies may assist in exploring their application for cancer treatment.

\section{Limitations of polyphenols as anticancer agent}

Polyphenolic compounds, which are abundant in dietary sources, show great promise in treating cancer, especially in consideration of their safe consumption. However, one of the major concern with using polyphenols as anticancer agents individually is their poor bioavailability in the human body. In addition, their interactions with other natural compounds in a diet may hinder or complicate consistency of their efficacy. Therefore, specifically designed combinations of several polyphenols or combinations of polyphenols with other natural agents aimed at defined biological targets will expand metabolic effects of the constituents of such mixtures in controlled and reproducible ways (14).

\section{Summary}

The desire for new successful drugs capable of battling cancer is still a challenge in medical science. Natural organisms (e.g., plants, bacteria, fungi) provide active molecules with a potential application in medicine for the management of several diseases, including cancer. The health effects of polyphenols depend upon both their 
respective intakes and bioavailability. The current review of some targeted polyphenol compounds (quercetin, curcumin, resveratrol, and kaempferol) and anticancer effects in various cancer cell lines is provided. The mode of action included mainly modulation of molecular events and signaling pathways linked to cell survival, proliferation, metastasis, apoptosis, and angiogenesis. Therefore, future research directions should extend the reach of natural compounds as healthy, safe, effective, and cost-effective therapeutic solutions to cancer. The in vitro and in vivo anticancer effects of reported polyphenol compounds are illustrated in Table 2.

\section{Acknowledgments}

Funding: This study was supported by the National Research Foundation of Korea funded by the Ministry of Science and ICT (grant nos. 2012M3A9B8019303 and 2020R1A2B5B01001807). PBB is supported by a global grant (GG1876852) from Rotary International.

\section{Footnote}

Reporting Checklist: The authors have completed the Narrative Review reporting checklist. Available at http:// dx.doi.org/10.21037/tcr-20-2359

Peer Review File: Available at http://dx.doi.org/10.21037/tcr20-2359

Conflicts of Interest: All authors have completed the ICMJE uniform disclosure form (available at http://dx.doi. org/10.21037/tcr-20-2359). The authors have no conflicts of interest to declare.

Ethical Statement: The authors are accountable for all aspects of the work in ensuring that questions related to the accuracy or integrity of any part of the work are appropriately investigated and resolved.

Open Access Statement: This is an Open Access article distributed in accordance with the Creative Commons Attribution-NonCommercial-NoDerivs 4.0 International License (CC BY-NC-ND 4.0), which permits the noncommercial replication and distribution of the article with the strict proviso that no changes or edits are made and the original work is properly cited (including links to both the formal publication through the relevant DOI and the license).
See: https://creativecommons.org/licenses/by-nc-nd/4.0/.

\section{References}

1. George VC, Dellaire G, Rupasinghe HV. Plant flavonoids in cancer chemoprevention: role in genome stability. J Nutr Biochem 2017;45:1-14.

2. Bray F, Ferlay J, Soerjomataram I, et al. Global cancer statistics 2018: GLOBOCAN estimates of incidence and mortality worldwide for 36 cancers in 185 countries. CA Cancer J Clin 2018;68:394-424.

3. Jemal A, Center MM, DeSantis C, et al. Global patterns of cancer incidence and mortality rates and trends. Cancer Epidemiol Biomarkers Prev 2010;19:1893-907.

4. Trosko JE. Evolution of energy metabolism, stem cells and cancer stem cells: how the Warburg and Barker hypothesis might be linked. BMC Proc 2013;7 Suppl 2:K8.

5. Block KI, Gyllenhaal C, Lowe L, et al. Designing a broadspectrum integrative approach for cancer prevention and treatment. Semin Cancer Biol 2015;35 Suppl:S276-304.

6. Lee HJ, Saralamma VVG, Kim SM, et al. Pectolinarigenin induced cell cycle arrest, autophagy, and apoptosis in gastric cancer cell via PI3K/AKT/mTOR signaling pathway. Nutrients 2018;10:1043.

7. Jabir NR, Anwar K, Firoz CK, et al. An overview on the current status of cancer nanomedicines. Curr Med Res Opin 2018;34:911-21.

8. Sharma P, McClees SF, Afaq F. Pomegranate for prevention and treatment of cancer: an update. Molecules 2017;22:177.

9. Rehan M, Mahmoud MM, Tabrez S, et al. Exploring flavonoids for potential inhibitors of a cancer signaling protein PI3K $\gamma$ kinase using computational methods. Anticancer Res 2020;40:4547-56.

10. Newman DJ, Cragg GM. Natural products as sources of new drugs over the last 25 years. J Nat Prod 2007;70:461-77.

11. Buyel JF. Plants as sources of natural and recombinant anti-cancer agents. Biotechnol Adv 2018;36:506-20.

12. Paz MF, Islam MT, Tabrez S, et al. Effect of diterpenes on hepatic system. Curr Pharm Des 2018;24:4093-100.

13. Asensi M, Ortega A, Mena S, et al. Natural polyphenols in cancer therapy. Crit Rev Clin Lab Sci 2011;48:197-216.

14. Niedzwiecki A, Roomi MW, Kalinovsky T, et al. Anticancer efficacy of polyphenols and their combinations. Nutrients 2016;8:552.

15. Abbas M, Saeed F, Anjum FM, et al. Natural polyphenols: an overview. Int J Food Prop 2017;20:1689-99. 
16. Spatafora C, Tringali C. Natural-derived polyphenols as potential anticancer agents. Anticancer Agents Med Chem 2012;12:902-18.

17. Manach C, Scalbert A, Morand C, et al. Polyphenols: food sources and bioavailability. Am J Clin Nutr 2004;79:727-47.

18. Bhat KP, Pezzuto JM. Cancer chemopreventive activity of resveratrol. Ann Ny Acad Sci 2002;957:210-29.

19. Adlercreutz H, Mazur W. Phyto-oestrogens and Western diseases. Ann Med 1997;29:95-120.

20. Grosso G, Godos J, Lamuela-Raventos R, et al. A comprehensive meta-analysis on dietary flavonoid and lignan intake and cancer risk: level of evidence and limitations. Mol Nutr Food Res 2017. doi: 10.1002/ mnfr.201600930.

21. Messina M. Impact of soy foods on the development of breast cancer and the prognosis of breast cancer patients. Forsch Komplementmed 2016;23:75-80.

22. Rothwell JA, Knaze V, Zamora-Ros R. Polyphenols: dietary assessment and role in the prevention of cancers. Curr Opin Clin Nutr Metab Care 2017;20:512-21.

23. Guo Y, Zhi F, Chen P, et al. Green tea and the risk of prostate cancer: a systematic review and meta-analysis. Medicine (Baltimore) 2017;96:e6426.

24. Christensen KY, Naidu A, Parent MÉ, et al. The risk of lung cancer related to dietary intake of flavonoids. Nutr Cancer 2012;64:964-74.

25. Tabrez S, Priyadarshini M, Urooj M, et al. Cancer chemoprevention by polyphenols and their potential application as nanomedicine. J Environ Sci Health C Environ Carcinog Ecotoxicol Rev 2013;31:67-98.

26. Massi A, Bortolini O, Ragno D, et al. Research progress in the modification of quercetin leading to anticancer agents. Molecules 2017;22:1270.

27. Tabrez S, Jabir NR, Adhami VM, et al. Nanoencapsulated dietary polyphenols for cancer prevention and treatment: successes and challenges. Nanomedicine (Lond) 2020;15:1147-62.

28. Reyes-Farias M, Carrasco-Pozo C. The anti-cancer effect of quercetin: molecular implications in cancer metabolism. Int J Mol Sci 2019;20:3177.

29. Kashyap D, Garg VK, Tuli HS, et al. Fisetin and quercetin: promising flavonoids with chemopreventive potential. Biomolecules 2019;9:174.

30. Andres S, Pevny S, Ziegenhagen R, et al. Safety aspects of the use of quercetin as a dietary supplement. Mol Nutr Food Res 2018;62:1700447.

31. Dai W, Gao Q, Qiu J, et al. Quercetin induces apoptosis and enhances 5-FU therapeutic efficacy in hepatocellular carcinoma. Tumour Biol 2016;37:6307-13.

32. Duo J, Ying GG, Wang GW, et al. Quercetin inhibits human breast cancer cell proliferation and induces apoptosis via Bcl-2 and Bax regulation. Mol Med Rep 2012;5:1453-6.

33. Kim HS, Wannatung T, Lee S, et al. Quercetin enhances hypoxia-mediated apoptosis via direct inhibition of AMPK activity in HCT116 colon cancer. Apoptosis 2012;17:938-49.

34. Wang P, Zhang K, Zhang Q, et al. Effects of quercetin on the apoptosis of the human gastric carcinoma cells. Toxicol In Vitro 2012;26:221-8.

35. Granato M, Rizzello C, Montani MS, et al. Quercetin induces apoptosis and autophagy in primary effusion lymphoma cells by inhibiting PI3K/AKT/mTOR and STAT3 signaling pathways. J Nutr Biochem 2017;41:124-36.

36. Wang Y, Zhang W, Lv Q, et al. The critical role of quercetin in autophagy and apoptosis in HeLa cells. Tumour Biol 2016;37:925-9.

37. Wu L, Li J, Liu T, et al. Quercetin shows anti-tumor effect in hepatocellular carcinoma LM3 cells by abrogating JAK2/STAT3 signaling pathway. Cancer Med 2019;8:4806-20.

38. Yu D, Ye T, Xiang Y, et al. Quercetin inhibits epithelialmesenchymal transition, decreases invasiveness and metastasis, and reverses IL-6 induced epithelialmesenchymal transition, expression of MMP by inhibiting STAT3 signaling in pancreatic cancer cells. Onco Targets Ther 2017;10:4719-29.

39. Kee JY, Han YH, Kim DS, et al. Inhibitory effect of quercetin on colorectal lung metastasis through inducing apoptosis, and suppression of metastatic ability. Phytomedicine 2016;23:1680-90.

40. Zlotogorski A, Dayan A, Dayan D, et al. Nutraceuticals as new treatment approaches for oral cancer--I: curcumin. Oral Oncol 2013;49:187-91.

41. Giordano A, Tommonaro G. Curcumin and cancer. Nutrients 2019;11:2376.

42. Akbik D, Ghadiri M, Chrzanowski W, et al. Curcumin as a wound healing agent. Life Sci 2014;116:1-7.

43. Devassy JG, Nwachukwu ID, Jones PJ. Curcumin and cancer: barriers to obtaining a health claim. Nutr Rev 2015;73:155-65.

44. Wang JB, Qi LL, Wu TX. Curcumin induces apoptosis through the mitochondria-mediated apoptotic pathway in HT-29 cells. J Zhejiang Univ Sci B 2009;10:93-102. 
45. Hussain AR, Al-Rasheed M, Manogaran PS, et al. Curcumin induces apoptosis via inhibition of PI3 on of PI3ochondria-mediated apoptoticleukemias. Apoptosis 2006;11:245-54.

46. Jin H, Qiao F, Wang Y, et al. Curcumin inhibits cell proliferation and induces apoptosis of human non-small cell lung cancer cells through the upregulation of miR192-5p and suppression of PI3K/Akt signaling pathway. Oncol Rep 2015;34:2782-9.

47. Liu LD, Pang YX, Zhao XR, et al. Curcumin induces apoptotic cell death and protective autophagy by inhibiting AKT/mTOR/p70S6K pathway in human ovarian cancer cells. Arch Gynecol Obstet 2019;299:1627-39.

48. Zhu J, Zhao B, Xiong P, et al. Curcumin induces autophagy via inhibition of yes-associated protein (YAP) in human colon cancer cells. Med Sci Monit 2018;24:7035-42.

49. Zhu Y, Bu S. Curcumin induces autophagy, apoptosis, and cell cycle arrest in human pancreatic cancer cells. Evid Based Complement Alternat Med 2017;2017:5787218.

50. Cao AL, Tang QF, Zhou WC, et al. Ras/ERK signaling pathway is involved in curcumin-induced cell cycle arrest and apoptosis in human gastric carcinoma AGS cells. J Asian Nat Prod Res 2015;17:56-63.

51. Yang J, Wang C, Zhang Z, et al. Curcumin inhibits the survival and metastasis of prostate cancer cells via the Notch-1 signaling pathway. APMIS 2017;125:134-40.

52. Mohankumar K, Sridharan S, Pajaniradje S, et al. BDMC-A, an analog of curcumin, inhibits markers of invasion, angiogenesis, and metastasis in breast cancer cells via NF- $\kappa \mathrm{B}$ pathway--a comparative study with curcumin. Biomed Pharmacother 2015;74:178-86.

53. Shankar S, Ganapathy S, Chen Q, et al. Curcumin sensitizes TRAIL-resistant xenografts: molecular mechanisms of apoptosis, metastasis and angiogenesis. Mol Cancer 2008;7:16.

54. Rauf A, Imran M, Butt MS, et al. Resveratrol as an anti-cancer agent: a review. Crit Rev Food Sci Nutr 2018;58:1428-47.

55. Ko JH, Sethi G, Um JY, et al. The role of resveratrol in cancer therapy. Int J Mol Sci 2017;18:2589.

56. Meng X, Zhou J, Zhao CN, et al. Health benefits and molecular mechanisms of resveratrol: a narrative review. Foods 2020;9:340.

57. Wang Z, Li W, Meng X, et al. Resveratrol induces gastric cancer cell apoptosis via reactive oxygen species, but independent of sirtuin1. Clin Exp Pharmacol Physiol 2012;39:227-32.
58. Kumar S, Eroglu E, Stokes III JA, et al. Resveratrol induces mitochondria-mediated, caspase-independent apoptosis in murine prostate cancer cells. Oncotarget 2017;8:20895.

59. Yu XD, Yang JL, Zhang WL, et al. Resveratrol inhibits oral squamous cell carcinoma through induction of apoptosis and G2/M phase cell cycle arrest. Tumour Biol 2016;37:2871-7.

60. Heo JR, Kim SM, Hwang KA, et al. Resveratrol induced reactive oxygen species and endoplasmic reticulum stress-mediated apoptosis, and cell cycle arrest in the A375SM malignant melanoma cell line. Int J Mol Med 2018;42:1427-35.

61. Zhang B, Yin X, Sui S. Resveratrol inhibited the progression of human hepatocellular carcinoma by inducing autophagy via regulating p 53 and the phosphoinositide 3-kinase/protein kinase B pathway. Oncol Rep 2018;40:2758-65.

62. Gong C, Xia H. Resveratrol suppresses melanoma growth by promoting autophagy through inhibiting the PI3K/AKT/mTOR signaling pathway. Exp Ther Med 2020;19:1878-86.

63. Garvin S, Öllinger K, Dabrosin C. Resveratrol induces apoptosis and inhibits angiogenesis in human breast cancer xenografts in vivo. Cancer Lett 2006;231:113-22.

64. Ren J, Lu Y, Qian Y, et al. Recent progress regarding kaempferol for the treatment of various diseases. Exp Ther Med 2019;18:2759-76.

65. Devi KP, Malar DS, Nabavi SF, et al. Kaempferol and inflammation: from chemistry to medicine. Pharmacol Res 2015;99:1-10

66. Imran M, Salehi B, Sharifi-Rad J, et al. Kaempferol: a key emphasis to its anticancer potential. Molecules 2019;24:2277.

67. Calderón-Montaño JM, Burgos-Morón E, Pérez-Guerrero $\mathrm{C}$, et al. A review on the dietary flavonoid kaempferol. Mini Rev Med Chem 2011;11:298-344.

68. Yang S, Si L, Jia Y, et al. Kaempferol exerts antiproliferative effects on human ovarian cancer cells by inducing apoptosis, G0/G1 cell cycle arrest and modulation of MEK/ERK and STAT3 pathways. J BUON 2019;24:975-81.

69. Lee HS, Cho HJ, Yu R, et al. Mechanisms underlying apoptosis-inducing effects of Kaempferol in HT-29 human colon cancer cells. Int J Mol Sci 2014;15:2722-37.

70. Kim TW, Lee SY, Kim M, et al. Kaempferol induces autophagic cell death via IRE1-JNK-CHOP pathway and inhibition of G9a in gastric cancer cells. Cell Death Dis 
2018;9:875.

71. Guo H, Lin W, Zhang X, et al. Kaempferol induces hepatocellular carcinoma cell death via endoplasmic reticulum stress-CHOP-autophagy signaling pathway. Oncotarget 2017;8:82207.

72. Cho HJ, Park JH. Kaempferol induces cell cycle arrest in HT-29 human colon cancer cells. J Cancer Prev 2013;18:257.

73. Choi EJ, Ahn WS. Kaempferol induced the apoptosis via cell cycle arrest in human breast cancer MDA-MB-453 cells. Nutr Res Pract 2008;2:322-5.

74. Yang F, Jiang X, Song L, et al. Quercetin inhibits angiogenesis through thrombospondin-1 upregulation to antagonize human prostate cancer PC-3 cell growth in vitro and in vivo. Oncol Rep 2016;35:1602-10.

75. Song $W, Z$ hao X, Xu J, et al. Quercetin inhibits angiogenesis-mediated human retinoblastoma growth by targeting vascular endothelial growth factor receptor. Oncol Lett 2017;14:3343-8.

76. Chen Y, Li XX, Xing NZ, et al. Quercetin inhibits choroidal and retinal angiogenesis in vitro. Graefes Arch Clin Exp Ophthalmol 2008;246:373-8.

77. Zhao X, Wang Q, Yang S, et al. Quercetin inhibits angiogenesis by targeting calcineurin in the xenograft model of human breast cancer. Eur J Pharmacol 2016;781:60-8.

78. Chen QY, Zheng Y, Jiao DM, et al. Curcumin inhibits lung cancer cell migration and invasion through Rac1-dependent signaling pathway. J Nutr Biochem 2014;25:177-85.

79. Zhang C, Hao Y, Wu L, et al. Curcumin induces apoptosis and inhibits angiogenesis in murine malignant mesothelioma. Int J Oncol 2018;53:2531-41.

80. Yang CL, Liu YY, Ma YG, et al. Curcumin blocks small cell lung cancer cells migration, invasion, angiogenesis, cell cycle and neoplasia through Janus kinase-STAT3

Cite this article as: Bhosale PB, Ha SE, Vetrivel P, Kim HH, Kim SM, Kim GS. Functions of polyphenols and its anticancer properties in biomedical research: a narrative review. Transl Cancer Res 2020;9(12):7619-7631. doi: 10.21037/tcr-20-2359 signalling pathway. PLoS One 2012;7:e37960.

81. Yeh CB, Hsieh MJ, Lin CW, et al. The antimetastatic effects of resveratrol on hepatocellular carcinoma through the downregulation of a metastasis-associated protease by SP-1 modulation. PLoS One 2013;8:e56661.

82. Yuan L, Zhou M, Huang D, et al. Resveratrol inhibits the invasion and metastasis of colon cancer through reversal of epithelial-mesenchymal transition via the AKT/GSK-3 / Snail signaling pathway. Mol Med Rep 2019;20:2783-95.

83. Fouad MA, Agha AM, Merzabani MA, et al. Resveratrol inhibits proliferation, angiogenesis and induces apoptosis in colon cancer cells: calorie restriction is the force to the cytotoxicity. Hum Exp Toxicol 2013;32:1067-80.

84. Lee SH, Koo BS, Park SY, et al. Anti-angiogenic effects of resveratrol in combination with 5-fluorouracil on B16 murine melanoma cells. Mol Med Rep 2015;12:2777-83.

85. Han X, Liu CF, Gao N, et al. Kaempferol suppresses proliferation but increases apoptosis and autophagy by upregulating microRNA-340 in human lung cancer cells. Biomed Pharmacother 2018;108:809-16.

86. Chen HJ, Lin CM, Lee CY, et al. Kaempferol suppresses cell metastasis via inhibition of the ERK-p38-JNK and AP-1 signaling pathways in U-2 OS human osteosarcoma cells. Oncol Rep 2013;30:925-32.

87. Lin CW, Chen PN, Chen MK, et al. Kaempferol reduces matrix metalloproteinase-2 expression by down-regulating ERK1/2 and the activator protein-1 signaling pathways in oral cancer cells. PLoS One 2013;8:e80883.

88. Luo H, Rankin GO, Liu L, et al. Kaempferol inhibits angiogenesis and VEGF expression through both HIF dependent and independent pathways in human ovarian cancer cells. Nutr Cancer 2009;61:554-63.

89. Qin Y, Cui W, Yang X, et al. Kaempferol inhibits the growth and metastasis of cholangiocarcinoma in vitro and in vivo. Acta Biochim Biophys Sin (Shanghai) 2016;48:238-45. 